Pacific Journal of Mathematics

ON THE RANGE OF A DERIVATION 


\section{ON THE RANGE OF A DERIVATION}

\section{J. P. Williams}

A derivation on an algebra $\mathscr{A}$ is a linear transformation $\delta$ on $\mathscr{A}$ with the property $\delta(X Y)=X \delta(Y)+\delta(X) Y$ for all $X, Y \in \mathscr{A}$. If $\mathscr{A}=\mathscr{B}(\mathscr{H})$ is the Banach algebra of all bounded linear operators on a complex separable infinitedimensional Hilbert space $\mathscr{H}$ then it is known that every derivation $\delta$ on $\mathscr{A}$ is inner, that is, there is a bounded operator $A$ on $\mathscr{H}$ such that $\delta(X)=A X-X A=\delta_{A}(X)$ for all $X \in \mathscr{B}(\mathscr{H})$. (See [8].) In the present note simple necessary and sufficient conditions are obtained that (i) the range $\mathscr{R}\left(\delta_{A}\right)$ be dense in the weak and ultraweak operator topologies; (ii) the norm closure of the range contain the ideal $\mathscr{K}$ of compact operators on $\mathscr{H}$, (iii) the set of commutators $B X-X B$ where $B$ belongs to the $C^{*}$-algebra generated by $A$ and $X$ is arbitrary be weakly or ultraweakly dense in $\mathscr{B}(\mathscr{H})$. The commutant of the range of a derivation is also computed and it is shown that the ranges of any two nonzero derivations have nonzero intersection.

1. If $A$ and $B$ are bounded operators on $\mathscr{H}$ then the identities $\delta_{A}+\delta_{B}=\delta_{A+B}, \delta_{A} \delta_{B}-\delta_{B} \delta_{A}=\delta_{A B-B A}$ show that the sum and Lie product of two (inner) derivations is a derivation. However the product $\delta_{A} \delta_{B}$ is a derivation only in the trivial cases:

Theorem 1. Let $A, B \in \mathscr{B}(\mathscr{H})$. The $\delta_{A} \delta_{B}$ is a derivation if and only if $A$ or $B$ is a scalar multiple of the identity operator.

Proof. Let $\delta=\delta_{A} \delta_{B}$. Then

$$
\begin{aligned}
\delta(X Y) & =\delta_{A}\left(\delta_{B}(X Y)\right)=\delta_{A}\left(X \delta_{B}(Y)+\delta_{B}(X) Y\right) \\
& \left.=X \delta_{A} \delta_{B}(Y)\right)+\delta_{A}(X) \delta_{B}(Y)+\delta_{A}\left(\delta_{B}(X)\right) Y+\delta_{B}(X) \delta_{A}(Y) \\
& =X \delta(Y)+\delta(X) Y+\delta_{A}(X) \delta_{B}(Y)+\delta_{B}(X) \delta_{A}(Y) .
\end{aligned}
$$

Therefore $\delta$ is a derivation if and only if

$$
\delta_{A}(X) \delta_{B}(Y)+\delta_{B}(X) \delta_{A}(Y)=0
$$

for all $X, Y \in \mathscr{B}(\mathscr{H})$. Replacing $X$ by $X Z$ in (1) we get

$$
0=X \delta_{A}(Z) \delta_{B}(Y)+\delta_{A}(X) Z \delta_{B}(Y)+X \delta_{B}(Z) \delta_{A}(Y)+\delta_{B}(X) Z \delta_{A}(Y)
$$

so that

$$
\delta_{A}(X) Z \delta_{B}(Y)+\delta_{B}(X) Z \delta_{A}(Y)=0
$$


for all $X, Y, Z \in \mathscr{B}(\mathscr{C})$. Now let $Z=\delta_{B}(W)$ in (2) and use (1) to get

$$
\begin{aligned}
0 & =\delta_{A}(X) \delta_{B}(W) \delta_{B}(Y)+\delta_{B}(X) \delta_{B}(W) \delta_{A}(Y) \\
& =-\delta_{B}(X) \delta_{A}(W) \delta_{B}(Y)+\delta_{B}(X) \delta_{B}(W) \delta_{A}(Y) \\
& =2 \delta_{B}(X) \delta_{B}(W) \delta_{A}(Y)
\end{aligned}
$$

Finally, replacing $X$ by $X V$ in (3) we get

$$
\begin{aligned}
0 & =X \delta_{B}(V) \delta_{B}(W) \delta_{A}(Y)+\delta_{B}(X) V \delta_{B}(W) \delta_{A}(Y) \\
& =\delta_{B}(X) V \delta_{B}(W) \delta_{A}(Y)
\end{aligned}
$$

Starting with (2) a similar argument gives

$$
0=\delta_{A}(X) V \delta_{A}(W) \delta_{B}(Y)
$$

Suppose now that $B$ is not a scalar multiple of the identity. Then there is an $X \in \mathscr{B}(\mathscr{C})$ for which $\delta_{B}(X) \neq 0$. The norm closed linear span $\mathscr{J}_{X}$ of the operators $\left\{V \delta_{B}(X) W: V, W \in \mathscr{B}(\mathscr{H})\right\}$ is a nonzero two-sided ideal in $\mathscr{B}(\mathscr{H})$, hence $\mathscr{F}_{X} \supset \mathscr{K}$. Hence by (4), $S \delta_{B}(Z) \delta_{A}(Y)=0$ for all $S \in \mathscr{K}$. Since multiplication is continuous in the weak operator topology and since $\mathscr{K}$ is dense in $\mathscr{B}(\mathscr{H})$ for this topology we conclude that

$$
\delta_{B}(Z) \delta_{A}(Y)=0
$$

for all $Z, Y \in \mathscr{B}(\mathscr{C})$.

Replacing $Z$ by $X Z$ in (5) we get

$$
\begin{aligned}
0 & =X \delta_{B}(Z) \delta_{A}(Y)+\delta_{B}(X) Z \delta_{A}(Y) \\
& =\delta_{B}(X) Z \delta_{A}(Y)
\end{aligned}
$$

for all $X, Y, Z \in \mathscr{P}(\mathscr{H})$. The preceding ideal argument then shows that $\delta_{A}(Y)=0$ for all $Y \in \mathscr{B}(\mathscr{C})$ so that $A$ is a scalar multiple of the identity.

A similar argument shows that $(4)^{\prime}$ implies

$$
\delta_{A}(Z) W \delta_{B}(Y)=0
$$

for all $W, Y, Z \in \mathscr{B}(\mathscr{H})$. It follows from (6)' that if $A$ is not a scalar multiple of the identity, then $B$ is.

In general the range of a non-invertible linear transformation is topologically small (first category) and has large codimension (see [5]). It is therefore of some interest to note that the range of a derivation is large in at least one sense, namely it must have a small commutant: 
CoRollary 1. If $A \in \mathscr{B}(\mathscr{H})$ is not a scalar multiple of the identity then $\mathscr{R}\left(\delta_{A}\right)^{\prime}=\boldsymbol{C}$.

Proof. Let $B \in \mathscr{R}\left(\delta_{A}\right)$. Then $\delta_{B}\left(\delta_{A}(X)\right)=0$ for all $X \in \mathscr{B}(\mathscr{H})$ so that $\delta_{B} \delta_{A}=0$ is a derivation. Hence $B$ is a scalar by the theorem.

The next result was conjectured by Joel Anderson. It too may be interpreted as additional evidence that derivations have large range.

CoRollary 2. If $A$ and $B$ are not scalar multiples of the identity then $\mathscr{R}\left(\delta_{A}\right) \cap \mathscr{R}\left(\delta_{B}\right) \neq 0$.

Proof. Suppose that $\mathscr{R}\left(\delta_{A}\right) \cap \mathscr{R}\left(\delta_{B}\right)=0$. Then $A B-B A=$ $\delta_{A}(B)=-\delta_{B}(A)$ belongs to the zero subspace so that $A B-B A=0$. Therefore $\delta=\delta_{A} \delta_{B}=\delta_{B} \delta_{A}$ has range consisting of 0 alone so that $A$ or $B$ must be a scalar by the theorem.

REMARKs. (1) The collection of ranges of (bounded) operators on $\mathscr{H}$ is a lattice with respect to intersection and vector sum (See $[4,5]$.$) However if A, B \in \mathscr{B}(\mathscr{H})$ then neither of the subspaces $\left.\mathscr{R}\left(\delta_{A}\right) \cap \mathscr{R}\left(\delta_{B}\right), \mathscr{R}\left(\delta_{A}\right)+\mathscr{R} \delta_{B}\right)$ need be the range of a derivation. Indeed, let $\mathscr{H}=\mathscr{L}_{1} \oplus \mathscr{H}_{2}$ be a decomposition of $\mathscr{H}$ into infinitedimensional subspaces and let $A=\left(\begin{array}{l}10 \\ 00\end{array}\right), B=\left(\begin{array}{l}00 \\ 10\end{array}\right)$ acting in the usual way on $\mathscr{H}_{1} \oplus \mathscr{H}_{2}$. A simple verification shows that if $T=\left(\begin{array}{l}P Q \\ R S\end{array}\right) \in \mathscr{B}(\mathscr{H})$ has the property $\mathscr{R}\left(\delta_{T}\right) \subset \mathscr{R}\left(\delta_{A}\right)+\mathscr{R}\left(\delta_{B}\right)$ then $P, Q, R, S$ are scalars. From this it is easy to see that $\mathscr{R}\left(\delta_{T}\right) \neq \mathscr{R}\left(\delta_{A}\right)+\mathscr{R}\left(\delta_{B}\right)$ and that $\mathscr{R}\left(\delta_{T}\right) \subset \mathscr{R}\left(\delta_{A}\right) \cap \mathscr{R}\left(\delta_{B}\right)$ only if $\delta_{T}=0$.

(2) The assertion of Corollary (2) is false for three derivations. In fact, if $A$ and $B$ are as in the preceding remark and if $C=B^{*}$ then $\mathscr{R}\left(\delta_{A}\right) \cap \mathscr{R}\left(\delta_{B}\right) \cap \mathscr{R}\left(\delta_{C}\right)=0$.

2. In the remainder of this note we shall be concerned with density properties of the range of a derivation on $\mathscr{B}(\mathscr{P})$. J. G. Stampfli [9] has shown that $\mathscr{R}\left(\delta_{A}\right)$ is never norm dense, so we consider the situation for the weak operator and weak* topologies on $\mathscr{B}(\mathscr{H})$.

Theorem 2. Let $A \in \mathscr{B}(\mathscr{H})$. Then $\mathscr{R}\left(\delta_{A}\right)$ is dense in the weak operator topology if and only if the commutant $\{A\}^{\prime}$ of $A$ does not contain a nonzero operator of finite rank.

Proof. If $\varphi$ is a linear functional on $\mathscr{B}(\mathscr{H})$ that is continuous in the weak operator topology then there are scalars $\lambda_{i} \geqq 0$ and 
orthonormal sets $\left\{e_{i}\right\}_{i=1}^{n},\left\{e_{i}^{\prime}\right\}_{\imath=1}^{n}$ such that

$$
\varphi(X)=\sum_{i=1}^{n} \lambda_{i}\left(X e_{i}, e_{\imath}^{\prime}\right)
$$

for all $X \in \mathscr{B}(\mathscr{H})$. (See [3; p. 39] for example.) With each such $\varphi$ we may associate the operator $F$ of finite rank given by $F=\sum_{i=1}^{n}$ $\lambda_{i}\left(e_{i} \otimes e_{i}^{\prime}\right)$. Here if $x, y \in \mathscr{H}$ then $x \otimes y$ is the rank one operator that takes $y$ into $x:(x \otimes y)(z)=(z, y) x$ for $z \in \mathscr{Z}$.

Now if $x, y \in \mathscr{H}$, then

$$
\begin{aligned}
((A F-F A) x, y) & =\left(\left(\sum_{i} \lambda_{i} A\left(e_{i} \otimes e_{i}^{\prime}\right)-\sum_{i} \lambda_{i}\left(e_{i} \otimes e_{i}^{\prime}\right) A\right) x, y\right) \\
& =\sum_{i} \lambda_{i}\left(x, e_{i}^{\prime}\right)\left(A e_{i}, y\right)-\sum_{i} \lambda_{i}\left(e_{i}, y\right)\left(A x, e_{i}^{\prime}\right) \\
& =\sum \lambda_{i}\left((x \otimes y) A e_{i}, e_{i}^{\prime}\right)-\sum \lambda_{i}\left(A(x \otimes y) e_{i}, e_{i}^{\prime}\right) \\
& =\varphi((x \otimes y) A-A(x \otimes y)) .
\end{aligned}
$$

The preceding computation shows that if $\varphi$ vanishes on the range of $\delta_{A}$ then $F$ commutes with $A$. Conversely, if $F$ commutes with $A$, then $\varphi(X A-A X)=0$ for all operators $X$ of finite rank, hence by weak continuity, $\varphi$ vanishes on the range of $\delta_{A}$.

REMARK. $\mathscr{R}\left(\delta_{A}\right)$ is dense in the weak operator topology if and only if it is dense in the strong operator topology because $\mathscr{B}(\mathscr{H})$ has the same continuous linear functionals for these topologies (See [3; p. 37].)

3. With respect to a suitable norm the space $\tau(\mathscr{H})$ of trace class operators on $\mathscr{\mathscr { C }}$ is a Banach space and $\mathscr{B}(\mathscr{H})$ is canonically identifiable with the conjugate space of $\tau(\mathscr{H})$. The weak* (or ultraweakly) continuous linear functionals are those of the form $X \rightarrow f_{T}(X)=\operatorname{tr}(X T)$ where $T \in \tau(\mathscr{H})$. The map $T \rightarrow f_{T}$ is an embedding of $\tau(\mathscr{C})$ into $\mathscr{B}(\mathscr{H})^{*}$ and the latter space splits into the direct sum $\mathscr{K}^{\circ} \oplus \tau(\mathscr{H})$ where $\mathscr{K}^{\circ}$ consists of those bounded linear functionals on $\mathscr{B}(\mathscr{C})$ that annihilate the compact operators. Thus each $f \in \mathscr{B}(\mathscr{H})^{*}$ has the form $f=f_{\circ}+f_{T}$ where $f_{T}$ is induced by a trace class operator as above and $f_{\circ} \in \mathscr{K}^{\circ}$. The sum is even orthogonal in the sense that $\|f\| \geqq\left\|f_{T}\right\|$. (See [3; p. 49].) We now show that the annihilator of the range of a derivation also splits into the direct sum of its parts in $\mathscr{K}^{\circ}$ and $\tau(\mathscr{C})$.

THEOREM 3. If $A \in \mathscr{B}(\mathscr{H})$, then

$$
\mathscr{R}\left(\delta_{A}\right)^{\circ}=\mathscr{R}\left(\delta_{A}\right)^{\circ} \cap \mathscr{K}^{\circ} \oplus\{A\}^{\prime} \cap \tau(\mathscr{H}) \text {. }
$$

Proof. Let $f$ be a norm-continuous linear functional that vanishes 
on $\mathscr{R}\left(\delta_{A}\right)$ and let $f=f_{\circ}+f_{T}$ where $f_{\circ} \in \mathscr{K}^{\circ}$ and $T \in \tau(\mathscr{H})$. If $x, y \in \mathscr{L}$ then

$$
\begin{aligned}
\operatorname{tr}\left(A x \otimes T^{*} y\right) & =f_{T}(A(x \otimes y))=f(A(x \otimes y))=f((x \otimes y) A) \\
& =f_{T}((x \otimes y) A)=\operatorname{tr}\left(x \otimes T^{*} A^{*} y\right) .
\end{aligned}
$$

Since $\operatorname{tr}(z \otimes W)=(z, w)$ it follows that

$$
\left(A x, T^{*} y\right)=\left(x, T^{*} A^{*} y\right)
$$

for all $x, y$ and hence $T$ commutes with $A$. Also $f_{T}(A X)=f_{T}(X A)$ for rank one, and therefore for finite rank operators $X$. Since these are weak* dense in $\mathscr{B}(\mathscr{H})$ it follows that $f_{T} \in \mathscr{R}\left(\delta_{A}\right)^{\circ}$. This implies $f_{\circ}=f-f_{T} \in \mathscr{R}\left(\delta_{A}\right)^{\circ}$.

Conversely, the preceding computation shows that if

$$
T \in\{A\}^{\prime} \cap \tau(\mathscr{H}),
$$

then $f_{T} \in \mathscr{R}\left(\delta_{A}\right)^{\circ}$.

COROLlaRY 1. Let $A \in \mathscr{B}(\mathscr{H})$. The following conditions are equivalent:

(1) The norm closure of $\mathscr{R}\left(\delta_{A}\right)$ contains $\mathscr{K}$.

(2) $R\left(\delta_{A}\right)$ is weak* dense in $\mathscr{B}(\mathscr{H})$.

(3) $\{A\}^{\prime} \cap \tau(\mathscr{H})=0$.

Proof. The negations of conditions (2) and (3) are each equivalent to the condition that $\mathscr{R}\left(\delta_{A}\right)^{\circ}$ contain a nonzero weak* continuous linear functional. By Theorem 3 this occurs if and only if $\mathscr{R}\left(\delta_{A}\right)^{\circ} \not \subset \mathscr{K}^{\circ}$. This last condition in turn is equivalent to the condition that $\mathscr{R}\left(\delta_{A}\right)^{-}$does not contain $\mathscr{K}$.

REMARKs. (1) It is easy to exhibit operators that do not commute with a nontrivial operator of trace class. For example the unilateral shift of multiplicity one does not commute with any nonzero compact operator. Hence there are many operators $A$ with the property that the norm closure of the range of $\delta_{A}$ contains the compact operators. However [2] there does not exist an operator $A$ such that $\mathscr{R}\left(\delta_{A}\right) \supset \mathscr{K}$. More generally [9], the range of a derivation can contain no nonzero two-sided ideal of $\mathscr{B}(\mathscr{H})$.

(2) In contrast with the preceding corollary, it is not known whether there is an operator $A$ such that the norm closure of $\mathscr{R}\left(\delta_{A}\right)$ contains the identity operator. (See [10]). Since $\mathscr{R}\left(\delta_{A}\right) \cap\{A\}^{\prime}$ is a two-sided ideal in $\{A\}^{\prime}$ this question asks for an operator $A$ such that $\mathscr{R}\left(\delta_{A}\right)^{-} \supset\{A\}^{\prime}$. Two results are of interest here, namely: $\mathscr{R}\left(\delta_{A}\right) \cap\{A\}^{\prime}$ 
consists of quasinilpotent operators (Kleinecke [6]), and: $\mathscr{R}\left(\delta_{A}\right)$ and $\{A\}^{\prime}$ are orthogonal if $A$ is an isometry or if $A$ is normal (Anderson [1]).

An operator $A$ on $\mathscr{H}$ belongs to the class $\mathscr{R}_{n}(1 \leqq n<\infty)$ if $A$ has an $n$-dimensional reducing subspace [7]. Fuglede's theorem implies that $A \in \bigcup_{n} \mathscr{R}_{n}$ if and only if $\{A\}^{\prime}$ contains a nonzero compact normal operator.

If $\mathscr{S}_{1}, \mathscr{S}_{2}$ are subsets of $\mathscr{B}(\mathscr{H})$, let $\left[\mathscr{S}_{1}, \mathscr{S}_{2}\right]$ denote the linear span of the commutators $S_{1} S_{2}-S_{2} S_{1}$ where $S_{i} \in \mathscr{S}_{i}(i=1,2)$. Also, let $C^{*}(A)$ denote the $C^{*}$-algebra generated by the operator $A$.

CoRollary 2. The following conditions are equivalent for $A \in \mathscr{B}(\mathscr{H}):$

(1) $A \in \cup \mathscr{R}_{n}$.

(2) $\{A\}^{\prime}$ contains a nonzero compact normal operator.

(3) $\left[C^{*}(A), \mathscr{B}(\mathscr{H})\right]$ is not weak* dense in $\mathscr{B}(\mathscr{H})$.

(4) $\left[C^{*}(A), \mathscr{B}(\mathscr{H})\right]$ is not dense in $\mathscr{B}(\mathscr{H})$ for the weak operator topology.

Proof. The subspace $\mathscr{S}=\left[C^{*}(A), \mathscr{B}(\mathscr{C})\right]$ is self-adjoint and therefore is not weak* dense if and only if there is a nonzero selfadjoint weak* continuous linear functional $f_{T}$ that vanishes on $\mathscr{S}$. Since $f_{T}$ is norm continuous and self-adjoint, $f_{T}$ vanishes on $\mathscr{S}$ if and only if $f_{T}$ vanishes on the range of $\delta_{A}$. (See [10; Theorem 4] for example.) Also, $f_{T}$ is self-adjoint if and only if $T^{*}=T$. Thus conditions (1) and (3) are equivalent by Theorem 3 .

Linear functionals that are continuous in the weak operator topology correspond (as in the proof of Theorem 2) to operators of finite rank, and so a similar argument shows that (1) and (4) are equivalent. Finally, we have already observed that (1) and (2) are equivalent.

REMARKs. (1) Corollary 2 of course remains true if $C^{*}(A)$ is replaced by the von Neumann algebra generated by $A$ in (3) and (4).

(2) If $T=T^{*} \in \tau(\mathscr{H})$ is not of finite rank then the null space of $f_{T}$ is weak* closed but weak operator topology dense in $\mathscr{B}(\mathscr{H})$. Thus the equivalence of (3) and (4) is not valid for arbitrary selfadjoint subspaces.

(3) An operator $A$ belongs to the class $\mathscr{F}$ of finite operators if there is a positive central linear functional for $A$. (By definition, $f \in \mathscr{B}(\mathscr{H})^{*}$ is central for $A$ if $f(A X)=f(X A)$ for all $X \in \mathscr{B}(\mathscr{H})$.) In [10] it was observed that $\bigcup_{n} \mathscr{R}_{n} \subset \mathscr{F}$ and conjectured that the union is norm dense in $\mathscr{F}$. This and the preceding corollary suggest 
another conjecture, namely: $A \in \mathscr{F}$ if and only if $\left[C^{*}(A), \mathscr{B}(\mathscr{H})\right]$ is not norm dense in $\mathscr{B}(\mathscr{H})$. Necessity was proved in [10]. To prove sufficiency one needs to show that if there is a self-adjoint central linear functional for $A$, then there is also a positive central functional for $A$.

\section{REFERENCES}

1. Joel Anderson, On normal derivations, to appear.

2. A. Brown and C. Pearcy, Compact restrictions of operators, to appear.

3. J. Dixmier, Les Algebres d'Opérateurs dans l'Espace Hilbertien, Gauthier Villars, 1957.

4. J. Dixmier, Étude sur les variétés et les opérateurs de Julia, Bull. Soc. Math., France 77 (1949), 11-101.

5. P. A. Fillmore, J. P. Williams, On operator ranges, Advances in Math., to appear. 6. D. C. Kleinecke, On operator commutators, Proc. Amer. Math. Soc., 8 (1957), $535-536$.

7. P. R. Halmos, Irreducible operators, Mich. Math. J., 15 (1968), 215-223.

8. R. V. Kadison, Derivations of operator algebras, Ann. of Math., 83 (1966), 280-293.

9. J. G. Stampfli, On the range of a derivation, to appear.

10. J. P. Williams, Finite operators, Proc. Amer. Math. Soc., 26 (1970), 129-136.

Received December 22, 1970. The author gratefully acknowledges the support of the National Science Foundation.

INDIANA UNIVERSITY 



\section{PACIFIC JOURNAL OF MATHEMATICS}

\section{EDITORS}

H. SAMELSON

Stanford University

Stanford, California 94305

C. R. Hовву

University of Washington

Seattle, Washington 98105
J. DUGUNDJI

Department of Mathematics

University of Southern California

Los Angeles, California 90007

RICHARD ARENS

University of California

Los Angeles, California 90024

\section{ASSOCIATE EDITORS}
E. F. BECKENBACH
B. H. NeumanN
F. WOLF
K. YoSHIDA

\section{SUPPORTING INSTITUTIONS}

UNIVERSITY OF BRITISH COLUMBIA

CALIFORNIA INSTITUTE OF TECHNOLOGY

UNIVERSITY OF CALIFORNIA

MONTANA STATE UNIVERSITY

UNIVERSITY OF NEVADA

NEW MEXICO STATE UNIVERSITY

OREGON STATE UNIVERSITY

UNIVERSITY OF OREGON

OSAKA UNIVERSITY

UNIVERSITY OF SOUTHERN CALIFORNIA
STANFORD UNIVERSITY

UNIVERSITY OF TOKYO

UNIVERSITY OF UTAH

WASHINGTON STATE UNIVERSITY

UNIVERSITY OF WASHINGTON

AMERICAN MATHEMATICAL SOCIETY CHEVRON RESEARCH CORPORATION NAVAL WEAPONS CENTER 


\section{Pacific Journal of Mathematics}

\section{Vol. 38, No. $1 \quad$ March, 1971}

Bruce Alan Barnes, Banach algebras which are ideals in a Banach algebra ..... 1

David W. Boyd, Inequalities for positive integral operators............... 9

Lawrence Gerald Brown, Note on the open mapping theorem .............. 25

Stephen Daniel Comer, Representations by algebras of sections over Boolean

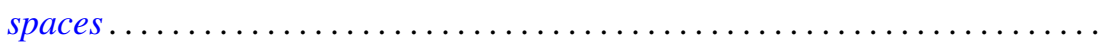

John R. Edwards and Stanley G. Wayment, On the nonequivalence of

conservative Hausdorff methods and Hausdorff moment sequences ........

P. D. T. A. Elliott, On the limiting distribution of additive functions $(\bmod 1) \ldots \ldots$

Mary Rodriguez Embry, Classifying special operators by means of subsets

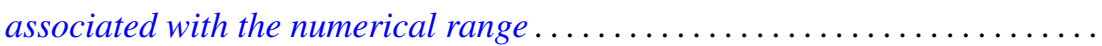

Darald Joe Hartfiel, Counterexamples to a conjecture of G. N. de Oliveira ......

C. Ward Henson, A family of countable homogeneous graphs...............

Satoru Igari and Shigehiko Kuratsubo, A sufficient condition for

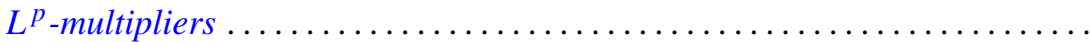

William A. Kirk, Fixed point theorems for nonlinear nonexpansive and

generalized contraction mappings............................

Erwin Kleinfeld, A generalization of commutative and associative rings ...... 95

D. B. Lahiri, Some restricted partition functions. Congruences modulo $11 \ldots \ldots 103$

T. Y. Lin, Homological algebra of stable homotopy ring $\pi *$ of spheres ....... 117

Morris Marden, A representation for the logarithmic derivative of a meromorphic function...........................

John Charles Nichols and James C. Smith, Examples concerning sum properties for metric-dependent dimension functions . .

Asit Baran Raha, On completely Hausdorff-completion of a completely

Hausdorff space.

M. Rajagopalan and Bertram Manuel Schreiber, Ergodic automorphisms and affine transformations of locally compact groups..........

N. V. Rao and Ashoke Kumar Roy, Linear isometries of some function

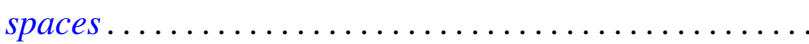

William Francis Reynolds, Blocks and F-class algebras of finite groups

Richard Rochberg, Which linear maps of the disk algebra are multiplicative ...

Gary Sampson, Sharp estimates of convolution transforms in terms of decreasing

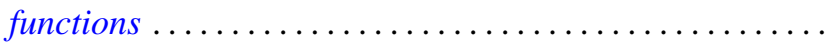

Stephen Scheinberg, Fatou's lemma in normed linear spaces

Ken Shaw, Whittaker constants for entire functions of several complex

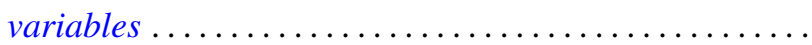

James DeWitt Stein, Two uniform boundedness theorems................ 251

$\mathrm{Li} \mathrm{Pi} \mathrm{Su,} \mathrm{Homomorphisms} \mathrm{of} \mathrm{near-rings} \mathrm{of} \mathrm{continuous} \mathrm{functions} \mathrm{.} \mathrm{.............} 261$

Stephen Willard, Functionally compact spaces, $C$-compact spaces and mappings of minimal Hausdorff spaces....................... 